\title{
INCLINATION ANGLE IMPLICATIONS FOR FLUID FLOW AND MIXED CONVECTION IN COMPLEX GEOMETRY ENCLOSURE-MESHLESS NUMERICAL ANALYSES
}

\author{
Mohammad NAJAFi \\ Department of Mechanical and Aerospace Engineering, Science and Research Branch, Islamic Azad University, Tehran, \\ Iran; e-mail: najafi@srbiau.ac.ir \\ MEHDi NIKFAR \\ Department of Mechanical Engineering, K.N. Toosi University of Technology, Tehran, Iran
}

Ali Arefmanesh

Department of Mechanical Engineering, University of Kashan, Kashan, Iran

\begin{abstract}
The meshless local Petrov-Galerkin (MLPG) method is extended to analyze the mixed convection and fluid flow in an inclined two-dimensional lid-driven cavity. The enclosure considered comprises two insulated vertical walls and a wavy bottom wall which is subjected to a higher constant temperature than its top counterpart, the sliding lid. For the proposed scheme, the stream function formulation with a weighting function of unity is employed. The simulation results reveal that the local Nusselt number increases with a clockwise increase in the inclination angle. Also, a decrease in the aspect ratio results in an increase in the hot wavy wall average Nusselt number.
\end{abstract}

Keywords: meshless, Petrov-Galerkin, mixed convection, rectangular cavity, wavy wall

\section{Introduction}

In the past two decades, a number of mesh-free numerical methods were proposed for solving partial differential equations. The principal objective of developing these methods was to get around the difficulties associated with generating a mesh in conventional numerical techniques such as the finite difference and the finite element methods. In the earlier meshfree techniques, an auxiliary grid had been required to evaluate the integrals resulting from applying the Galerkin method to the differential equations. Hence, these methods could not be considered totally meshless. Subsequently, two truly meshless techniques, the meshless local boundary equation (MLBE) method and the meshless local Petrov-Galerkin (MLPG) method were proposed by Zhu et al. (2004), and Atluri and Zhu (1998), respectively. To obtain the discretized equations in the latter scheme, instead of a mesh, an arbitrary point-distribution, without any pre-specified relationship among the points, is generated in the computational domain. Subsequently, the shape functions from the moving least squares (MLS) interpolations are employed in a local weak form of the differential equations over a local subdomain. The arbitrariness of the point-distribution as well as the possibility of adding or removing points to or from pre-existing point-distributions without any considerable computational effort make the MLPG method quite appealing for domains with complex geometries such as enclosures having wavy walls.

As far as application of the MLPG method on the fluid flow and heat transfer analysis, a number of recent studies may be mentioned. Lin and Atluri $(2001,2001)$ applied the newly-developed MLPG method to the solutions of the convection-diffusion and the Navier-Stokes (N-S) equations. They modified the local weak forms to overcome the so-called Babuska-Brezzi 
conditions while solving the primitive variables form of the N-S equations. Moreover, they presented a different upwinding scheme in order to obtain stabilized solutions under high Peclet and Reynolds (Re) numbers. Arefmanesh et al. (2005) applied a variation of the MLPG method with unity as the test function to the convection-diffusion and the potential flow equations. Comparisons of their results with the analytical solution for the convection-diffusion equation in a square domain demonstrated high accuracy of their proposed method. More recently, Arefmanesh et al. (2008) used a variation of the MLPG method with the unit test function to solve a number of non-isothermal fluid flow problems. They employed the stream function-vorticity formulation to solve different test cases such as a non-isothermal lid-driven cavity flow with an inlet and an outlet. In another study, Haji Mohammadi (2008) applied the MLPG method to simulate the incompressible viscous fluid flow. He formulated his considered cases in terms of the stream function-vorticity and employed the radial basis function interpolations in his approach.

Mixed convection flow and heat transfer in an enclosure involving a moving lid has drawn extensive attention due to its broad applications in engineering, modern technology, and natural situations. Among these applications, there is cooling of electronic devices, float glass production, food processing, and fluid flow and heat transfer in solar ponds. Flow and heat transfer from irregular surfaces are often encountered in many engineering applications to enhance heat transfer such as micro-electronic devices, solar collectors, electrical machinery, etc. Moreover, roughened and wavy surfaces could be used in the cooling of electrical and nuclear components to enhance the heat transfer. On the other hand, the study of natural and mixed convection heat transfer in inclined enclosures is relevant in geophysical situations, honeycomb solar collector plates, heat exchangers and crystal growth processes from melts, etc. Determining the optimal inclination angle is of paramount importance in such cases. As far as fundamental research is concerned, tilting the enclosure allows one to study different instabilities and their corresponding interactions through making a suitable choice of the values of the external parameters. Many recent studies regarding the applications of the conventional numerical methods on mixed convection are available in the literature. Chamkha (2002) conducted a numerical simulation of unsteady and laminar mixed convection heat transfer in a square cavity in the presence of internal heat generation or absorption and a magnetic field using the finite volume method. Mixed convection heat transfer in a two-dimensional rectangular cavity consisting of isothermal side walls moving vertically and a partially-heated bottom wall was studied numerically by Guo and Sharif (2004) using the finite volume method. In another study in the same year, Oztop and Dagtekin (2004) numerically simulated a steady state two-dimensional mixed convection problem in a cavity having two differentially-heated and vertically driven side walls using the finite volume method and the SIMPLE algorithm. The study showed that both the Richardson number and the direction of the moving walls affect the fluid flow and heat transfer inside the cavity. More recently, a finite element analysis of unsteady laminar mixed convection heat transfer in a cavity with sinusoidally oscillating horizontal top-lid was carried out by Khanafer et al. (2007). The laminar mixed convection heat transfer in two-dimensional shallow rectangular driven cavities with an aspect ratio of 10 was studied numerically by Sharif (2007) using the FLUENT version 6 commercial code. In another study, Al-Amiri et al. (2007) investigated the mixed convection heat transfer and fluid flow in a lid-driven cavity being heated from a sinusoidal wavy bottom surface using the finite element method. Their results showed that the average Nusselt number increased with an increase in both the amplitude of the wavy surface and the Reynolds number. Very recently, Nasrin (2012) conducted a finite element simulation of the mixed convection in an enclosure with a wavy bottom wall maintained at a higher temperature than those of the lid and two insulated side walls. For $\mathrm{Ra}=10^{4}$, $\mathrm{Re}=100$, and $\mathrm{Pr}=0.7$, Nasrin investigated the effects of the cavity aspect ratio, amplitude of the wavy wall and the number of undulations on the heat transfer inside the enclosure. It was concluded that increasing the wavy surface amplitude and cavity aspect ratio improved the heat transfer. 
In view of the above literature survey, there is not any type of meshless numerical treatment in the literature which would be applied to the mixed convection heat transfer in an irregular geometry enclosure with a wavy bottom wall within which the flow is induced by a shear force resulting from motion of the cavity lid combined with a buoyancy force resulting from the differentially-heated cavity walls. Moreover, a comprehensive study of the mixed convection in such an enclosure for a wide range of the Grashof and Richardson numbers as well as different inclination angles remains to be carried out. In the present study, therefore, the meshless local Petrov-Galerkin method is extended to analyze the mixed convection heat transfer in a lid-driven cavity with a wavy bottom wall and the effects of the inclination angle, Grashof number, Richardson number and the aspect ratio of the cavity on the fluid flow and heat transfer are investigated.

\section{Problem formulation}

A mixed convection fluid flow and heat transfer within a lid-driven rectangular cavity with a wavy bottom wall is simulated numerically using the MLPG method. As depicted in Fig. 1, the height and the width of the cavity are denoted by $H$ and $L$, respectively, and the aspect ratio of the cavity is defined as $A=H / L$. The left and the right walls of the cavity are insulated while the bottom wall of the enclosure, which is a wavy wall, is maintained at a constant temperature $T_{h}$, here referred to as "hot" temperature. The enclosure's top wall, which moves in its own plane from left to right with a constant speed $u_{l}$, is kept at a constant temperature $T_{c}$, here referred to as "cold" temperature, where $T_{h}>T_{c}$. The cavity is filled with a fluid with $\operatorname{Pr}=1$. The thermo-physical properties of the fluid are considered to be constant with the exception of density in the buoyancy term which, as will be noticed, varies according to the Bousinesq approximation (Bejan, 2004).

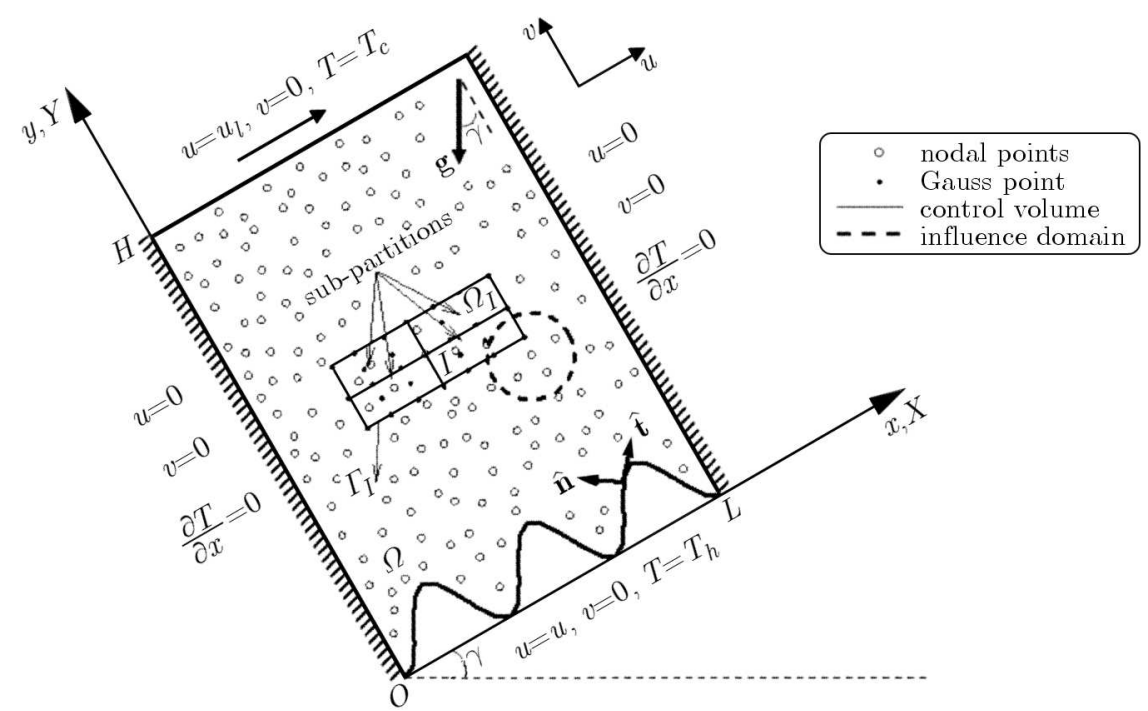

Fig. 1. Lid-driven differentially heated cavity, domain, boundary conditions and a typical control volume

The steady-state fluid flow and heat transfer in the cavity are governed by the continuity, momentum and energy equations. The natural convection term is incorporated in the momentum equation by employing the Bousinesq approximation (Bejan, 2004). In this study, the two-dimensional fluid flow problem is formulated in terms of the stream function and vorticity.

To cast the governing equations into a dimensionless form, the following dimensionless variables are introduced 


$$
\begin{aligned}
& X=\frac{x}{H} \quad Y=\frac{y}{H} \\
& U=\frac{u}{u_{l}} \quad V=\frac{v}{u_{l}} \quad \theta=\frac{T-T_{c}}{T_{h}-T_{c}} \\
& \Psi=\frac{\psi}{H u_{l}} \quad \Omega=\frac{\omega H}{u_{l}}
\end{aligned}
$$

where $u$ and $v$ are the velocity components in the $x$ and $y$-directions, respectively, $\psi$ is the stream function and $\omega$ is the vorticity. Substituting the above dimensionless variables into the stream function, vorticity and energy equations results in the following dimensionless form of the governing equations

$$
\begin{aligned}
& \frac{\partial^{2} \Psi}{\partial X^{2}}+\frac{\partial^{2} \Psi}{\partial Y^{2}}=-\Omega \\
& U \frac{\partial \Omega}{\partial X}+V \frac{\partial \Omega}{\partial Y}=\frac{1}{\operatorname{Re}}\left(\frac{\partial^{2} \Omega}{\partial X^{2}}+\frac{\partial^{2} \Omega}{\partial Y^{2}}\right)+\operatorname{Ri}\left(\cos \gamma \frac{\partial \theta}{\partial X}-\sin \gamma \frac{\partial \theta}{\partial Y}\right) \\
& U \frac{\partial \theta}{\partial X}+V \frac{\partial \theta}{\partial Y}=\frac{1}{\operatorname{RePr}}\left(\frac{\partial^{2} \theta}{\partial X^{2}}+\frac{\partial^{2} \theta}{\partial Y^{2}}\right)
\end{aligned}
$$

where $\gamma$ is the inclination angle of the cavity (Fig. 1). The Reynolds number Re and the Richardson number Ri are defined as

$$
\mathrm{Re}=\frac{u_{l} H}{\nu} \quad \mathrm{Ri}=\frac{\mathrm{Gr}}{\mathrm{Re}^{2}}
$$

where

$$
\mathrm{Gr}=\frac{g \beta\left(T_{h}-T_{c}\right) H^{3}}{\nu^{2}}
$$

where $\nu$ is the kinematic viscosity of the fluid, Gr is the Grashof number, and $\beta$ is the thermal expansion coefficient. The rate of heat transfer in the enclosure is obtained from the Nusselt number calculations. On the hot and the cold walls of the cavity, the local Nusselt number $(\mathrm{Nu})$ is obtained from the following relation

$$
\mathrm{Nu}=-\frac{\partial \theta}{\partial n}
$$

The Grashof numbers used in this study are $10^{4}$ and $10^{5}$. Two configurations for tilting the horizontal cavity, namely, clockwise and counter-clockwise about the origin, point $O$ (Fig. 1), are considered in this work. For each configuration, the inclination angle $\gamma$ is taken as $0,30^{\circ}$, $60^{\circ}$, and $90^{\circ}$. Also, for $\gamma=0$, the simulation is carried out for three aspect ratios of $A=0.5,1$, and 2 .

\section{Numerical procedure}

In the present study, the governing equations are solved numerically by the MLPG method. To implement the method, an arbitrary collection of points is selected in the computational domain (Fig. 1). Subsequently, a control volume is generated around each of the points. A typical rectangular control volume $\Omega_{I}$ generated around point $I$ is shown in Fig. 1 .

As the first step in developing the discretized equations for the control volume $\Omega_{I}$, the weak forms of the governing equations are to be obtained. This is accomplished by multiplying the stream function, vorticity and energy equations by the test function $W_{I}$. Subsequently, the resulting equations are integrated over $\Omega_{I}$. After performing the integration by parts, the weak 
forms of the stream function, vorticity and energy equations for the control volume $\Omega_{I}$ are expressed respectively

$$
\begin{aligned}
& -\int_{\Omega_{I}} \nabla W_{I} \cdot \nabla \Psi d \Omega+\int_{\Gamma_{I}-\Gamma_{I} \cap \Gamma_{h}} W_{I} \frac{\partial \Psi}{\partial n} d \Gamma+\int_{\Gamma_{I} \cap \Gamma_{h}} W_{I} \frac{\partial \Psi}{\partial n} d \Gamma=-\int_{\Omega_{I}} W_{I} \Omega d \Omega \\
& \int_{\Omega_{I}} W_{I}\left(U \frac{\partial \Omega}{\partial X}+V \frac{\partial \Omega}{\partial Y}\right) d \Omega=-\frac{1}{\operatorname{Re}} \int_{\Omega_{I}} \nabla W_{I} \cdot \nabla \Omega d \Omega+\frac{1}{\operatorname{Re}} \int_{\Gamma_{I}-\Gamma_{I} \cap \Gamma_{h}} W_{I} \frac{\partial \Omega}{\partial n} d \Gamma \\
& \quad+\frac{1}{\operatorname{Re}} \int_{\Gamma_{I} \cap \Gamma_{h}} W_{I} \frac{\partial \Omega}{\partial n} d \Gamma+\operatorname{Ri} \int_{\Omega_{I}} W_{I}\left(\frac{\partial \theta}{\partial X} \cos \gamma-\frac{\partial \theta}{\partial Y} \sin \gamma\right) d \Omega \\
& \int_{\left.\Omega_{I}\right)} W_{I}\left(U \frac{\partial \theta}{\partial X}+V \frac{\partial \theta}{\partial Y}\right) d \Omega=-\frac{1}{\operatorname{RePr}} \int_{\Omega_{I}} \nabla W_{I} \cdot \nabla \theta d \Omega+\frac{1}{\operatorname{RePr}} \int_{\Gamma_{I}-\Gamma_{I} \cap \Gamma_{h}} W_{I} \frac{\partial \theta}{\partial n} d \Gamma \\
& \quad+\frac{1}{\operatorname{RePr}} \int_{\Gamma_{I} \cap \Gamma_{h}} W_{I} \frac{\partial \theta}{\partial n} d \Gamma
\end{aligned}
$$

where $\Gamma_{h}$ is the portion of the domain boundary for which a natural boundary condition is enforced. Here, $\Gamma_{I}$ is the boundary of the control volume $\Omega_{I}$, and $\Gamma_{I} \cap \Gamma_{h}$ represents the intersection of $\Gamma_{I}$ with $\Gamma_{h}$.

Next, the integrals in Eqs. (3.1) are to be evaluated. The Gaussian quadrature is employed for this purpose. To perform the numerical integrations, the control volume $\Omega_{I}$ is divided into a number of sub-partitions (Fig. 1). The proper number of the Gauss points for the surface and the contour integrations in each sub-domain are selected. Subsequently, the field variables are approximated at each of the Gauss points using the MLS interpolations. Considering $\Omega_{k}$ as the interpolation domain for a typical Gauss point $k$, and $S_{j, j}=1(1) n_{k}$, as a collection of $n_{k}$ nodal points with coordinates $x_{j} \in \Omega_{k}$ (whose influence domains cover the considered Gauss point), the unknown stream function, vorticity and temperature fields are approximated within $\Omega_{k}$, respectively (Onate et al., 1996),

$$
\begin{aligned}
& \bar{\Psi}^{(k)}(\mathbf{x})=\sum_{j=1}^{n_{k}} N_{j}^{(k)}(\mathbf{x}) \widehat{\Psi}_{j} \quad \bar{\Omega}^{(k)}(\mathbf{x})=\sum_{j=1}^{n_{k}} N_{j}^{(k)}(\mathbf{x}) \widehat{\Omega}_{j} \\
& \bar{\theta}^{(k)}(\mathbf{x})=\sum_{j=1}^{n_{k}} N_{j}^{(k)}(\mathbf{x}) \widehat{\theta}_{j}
\end{aligned}
$$

where $\widehat{\Psi}_{j}, \widehat{\Omega}_{j}$, and $\widehat{\theta}_{j}$ are fictitious nodal values for the stream function, vorticity, and temperature, respectively. In Eqs. $(3.2), N_{j}^{(k)}(\mathbf{x}), j=1(1) n_{k}$, are the MLS interpolation functions (Onate et al., 1996).

Having obtained the weak formulations of the governing equations and the moving least-squares approximations of the field variables, the final step in the discretization process is to present the fully-discretized equations for a typical control volume $\Omega_{I}$ with the boundary $\Gamma_{I}$. For this purpose, the control volume $\Omega_{I}$ is divided into a number of sub-domains. The proper number of the Gauss points is selected in each of the sub-domains. Subsequently, the MLS interpolations for the stream function, vorticity and temperature (Eqs. (3.2)) at a typical Gauss point $k$ are substituted into Eqs. (3.1). The resulting discretized equations are given as 


$$
\begin{aligned}
& \sum_{j=1}^{n_{k}}\left(\int_{\Gamma_{I}-\Gamma_{I} \cap \Gamma_{h}} W_{I} N_{j, n}^{(k)}(\mathbf{x}) d \Gamma\right) \widehat{\Psi}_{j}=-\sum_{j=1}^{n_{k}}\left(\int_{\Omega_{I}} W_{I} N_{j}^{(k)}(\mathbf{x}) d \Omega\right) \widehat{\Omega}_{j} \\
& \quad-\int_{\Gamma_{I} \cap \Gamma_{h}} W_{I} \frac{\partial \Psi}{\partial n} d \Gamma+\sum_{j=1}^{n_{k}}\left(\int_{\Omega_{I}}\left(W_{I, X} N_{j, X}^{(k)}(\mathbf{x})+W_{I, Y} N_{j, Y}^{(k)}(\mathbf{x})\right) d \Omega\right) \widehat{\Psi}_{j} \\
& \sum_{j=1}^{n_{k}}\left(\int_{\Omega_{I}} W_{I}\left(U N_{j, X}^{(k)}(\mathbf{x})+V N_{j, Y}^{(k)}(\mathbf{x})\right) d \Omega-\frac{1}{\operatorname{Re}} \int_{\Gamma_{I}-\Gamma_{I} \cap \Gamma_{h}} W_{I} N_{j, n}^{(k)}(\mathbf{x}) d \Gamma\right) \widehat{\Omega}_{j} \\
& \quad+\frac{1}{\operatorname{Re}} \sum_{j=1}^{n_{k}}\left(\int_{\Omega_{I}}\left(W_{I, X} N_{j, X}^{(k)}(\mathbf{x})+W_{I, Y} N_{j, Y}^{(k)}(\mathbf{x})\right) d \Omega\right) \widehat{\Omega}_{j}=\frac{1}{\operatorname{Re}} \int_{\Gamma_{I} \cap \Gamma_{h}} W_{I} \frac{\partial \Omega}{\partial n} d \Gamma \\
& \quad+\operatorname{Ri} \sum_{j=1}^{n_{k}}\left(\int_{\Omega_{I}} W_{I}\left(N_{j, X}^{(k)}(\mathbf{x}) \cos \gamma-N_{j, Y}^{(k)}(\mathbf{x}) \sin \gamma\right) d \Omega\right) \widehat{\theta}_{j} \\
& \sum_{j=1}^{n_{k}}\left(\int_{\Omega_{I}} W_{I}\left(U N_{j, X}^{(k)}(\mathbf{x})+V N_{j, Y}^{(k)}(\mathbf{x})\right) d \Omega-\frac{1}{\operatorname{RePr}} \int_{\Gamma_{I}-\Gamma_{I} \cap \Gamma_{h}} W_{I} N_{j, n}^{(k)}(\mathbf{x}) d \Gamma\right) \widehat{\theta}_{j} \\
& \quad+\frac{1}{\operatorname{RePr}} \sum_{j=1}^{n_{k}}\left(\int_{\Omega_{I}}\left(W_{I, X} N_{j, X}^{(k)}(\mathbf{x})+W_{I, Y} N_{j, Y}^{(k)}(\mathbf{x})\right) d \Omega\right) \widehat{\theta}_{j}=\frac{1}{\operatorname{RePr}} \int_{\Gamma_{I} \cap \Gamma_{h}} W_{I} \frac{\partial \theta}{\partial n} d \Gamma
\end{aligned}
$$

The weighting function $W_{I}$ is taken to be unity $\left(W_{I}=1\right)$ in the present study.

Similar discretized equations are obtained for all the Gauss points within the control volume $\Omega_{I}$ and, in turn, for all the control volumes of the domain. Assembling these equations for the control volumes and enforcing the essential boundary conditions by the direct interpolation method yield a set of algebraic equations for the stream function, vorticity and temperature. Solving this system of algebraic equations using a band solver and the Gaussian elimination process yields the unknown values of the field variables at the points.

\section{Benchmarking of the code}

In order to validate the numerical procedure, the fluid flow and heat transfer inside a square cavity is solved using the present method and the obtained results are compared with those of Moallemi and Jang (1992) who used the FVM. The left and the right walls of the considered cavity are insulated and the enclosure's top and bottom walls are maintained at constant temperatures $T_{c}$ and $T_{h}$, respectively, with $T_{h}>T_{c}$. The top wall of the cavity moves in its own plane from the left to the right with a constant speed $U_{l}$. The simulations are performed for $\operatorname{Pr}=1$. Figure 2 shows the streamlines and the isotherms obtained by the MLPG simulation for $\mathrm{Ri}=1\left(\mathrm{Gr}=10^{6}, \mathrm{Re}=1000\right)$, and $\mathrm{Pr}=1$. The results presented by Moallemi and Jang (1992) using the FVM are also shown in this figure. As it can be seen from Fig. 2, very good agreements exist between the streamlines and the isotherms obtained by the MLPG simulation and those by Moallemi and Jang (1992). Comparisons between the local Nusselt numbers for the cold and the hot walls of the cavity obtained by the proposed MLPG method for $\operatorname{Pr}=1$, and the results of Moallemi and Jang (1992) are presented in Fig. 3. As it is observed from this figure, excellent agreements exist between the local Nusselt numbers for the two methods. 
(a)

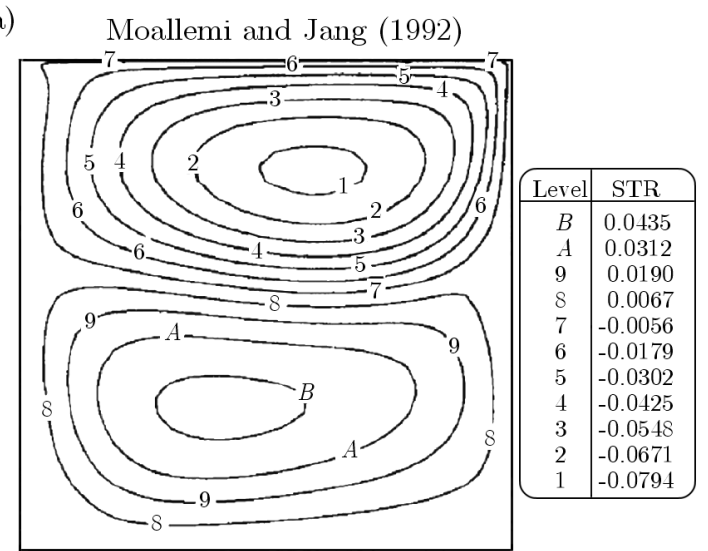

(b)

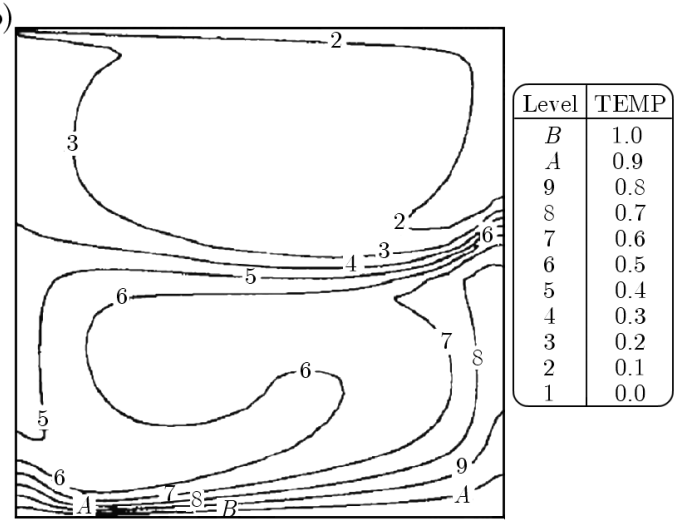

MPLG method
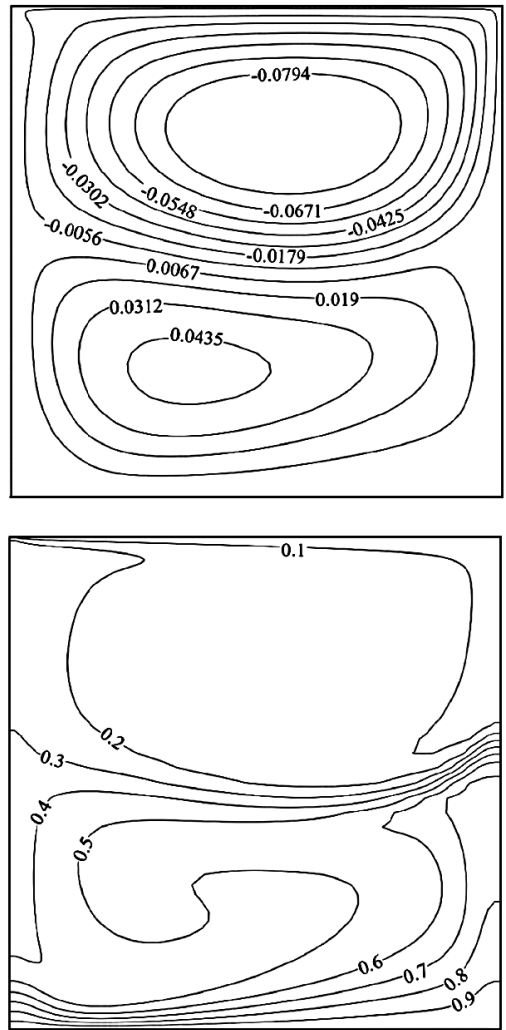

Fig. 2. Streamlines and isotherms, comparison between the MLPG method and the results of Moallemi and Jang (1992) for $\mathrm{Ri}=1,\left(\mathrm{Gr}=10^{6}, \mathrm{Re}=1000\right), \operatorname{Pr}=1$; (a) streamlines, (b) isotherms

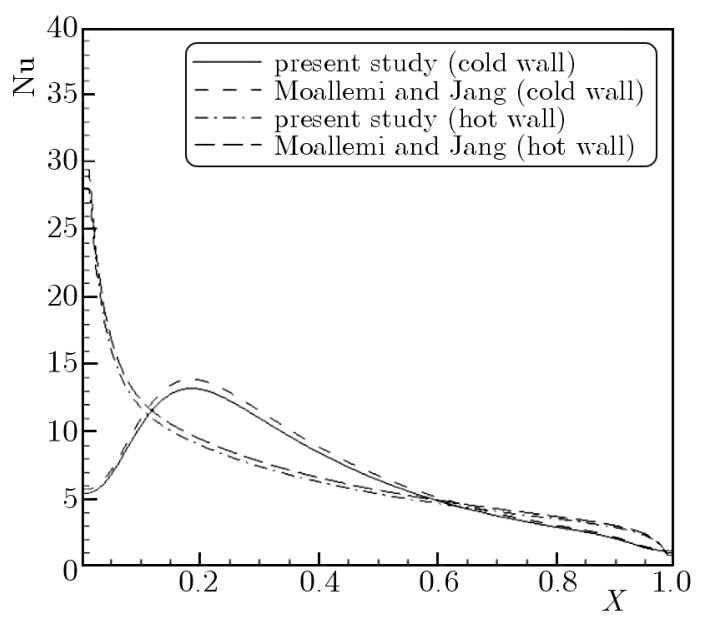

Fig. 3. Local Nusselt numbers, comparisons between the MLPG method and the results of Moallemi and Jang (1992) for $\mathrm{Ri}=1,\left(\mathrm{Gr}=10^{6}, \mathrm{Re}=1000\right)$, and $\operatorname{Pr}=1$

\section{Results and discussions}

Having validated the numerical scheme, the proposed code is employed to investigate the characteristics of the mixed convection heat transfer in rectangular cavities having wavy bottom walls (Fig. 1). The geometry of the wavy surface analyzed in this study is described by $Y=0.05(1-\cos (6 \pi X))$, where $X$ and $Y$ are the dimensionless Cartesian coordinates as shown in Fig. 1. The calculations are performed for a fluid with $\operatorname{Pr}=1$, for $\mathrm{Gr}=10^{4}$ and $10^{5}$, and $\mathrm{Ri}=1$. 
To analyze the effects of the inclination angle of the cavity $\gamma$ on the fluid flow and heat transfer, the simulations are performed for two configurations of tilting the cavity clockwise and counter-clockwise directions about point $O$ in Fig. 1. For each of the two configurations, the inclination angles of $0^{\circ}, 30^{\circ}, 60^{\circ}$, and $90^{\circ}$ are considered. The results discussed here are for $A=1, \mathrm{Ri}=1$ and 0.01 , and $\mathrm{Gr}=10^{4}$.

Figure 4 shows the streamlines and the isotherms inside the cavity for $\mathrm{Ri}=1$ and for different inclination angles obtained by tilting the cavity counter-clockwise about point $O$ (Fig. 1).The natural convection from the wavy hot wall intensifies with the increasing inclination angle. This, in turn, enhances the downstream eddy (DSE), and results in a relatively large counter-clockwise rotating vortex taking place in the lower portion of the cavity in the vicinity of the wavy wall with its illuminating eye coming closer to the cavity center away from the hot wavy wall. Consequently, the primary recirculation vortex weakens, and diminishes in size (Fig. 4a). The effect of the natural convection opposing the direction of the driven lid is more prominent in the vicinity of the wavy wall right edge, therefore, as it can be seen from Fig. 4b, the temperature gradients along the cold and a significant portion of the hot wall reduce substantially with the increasing inclination angle.
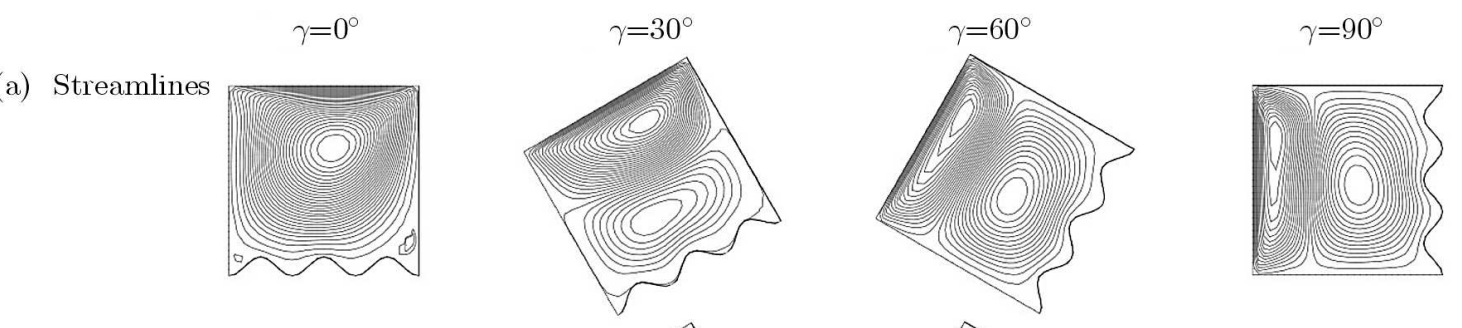

(b) Isotherms
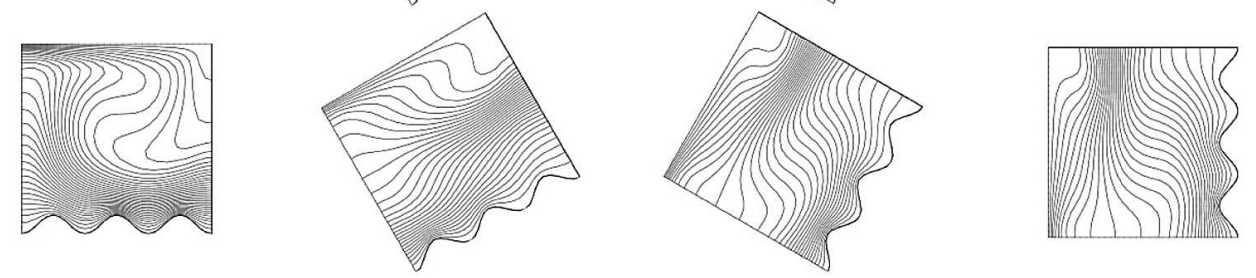

Fig. 4. Streamlines and isotherms for different counter-clockwise inclination angles, $\operatorname{Ri}=1, \mathrm{Gr}=10^{4}$, and $A=1$; (a) streamlines, (b) isotherms

(a)

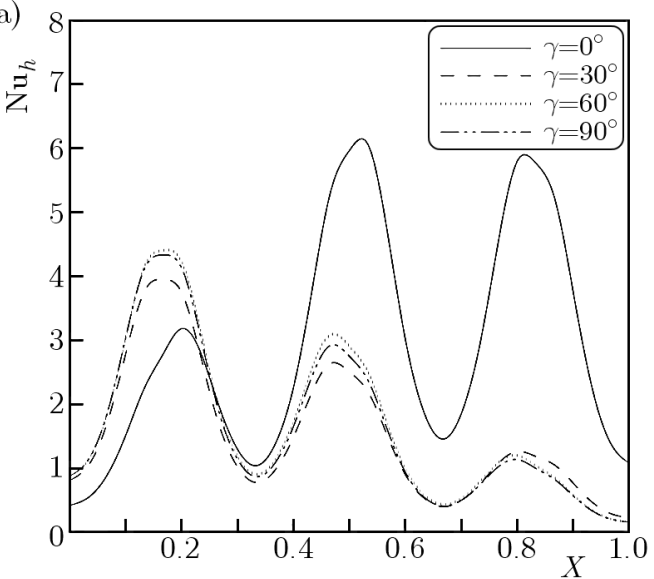

(b)

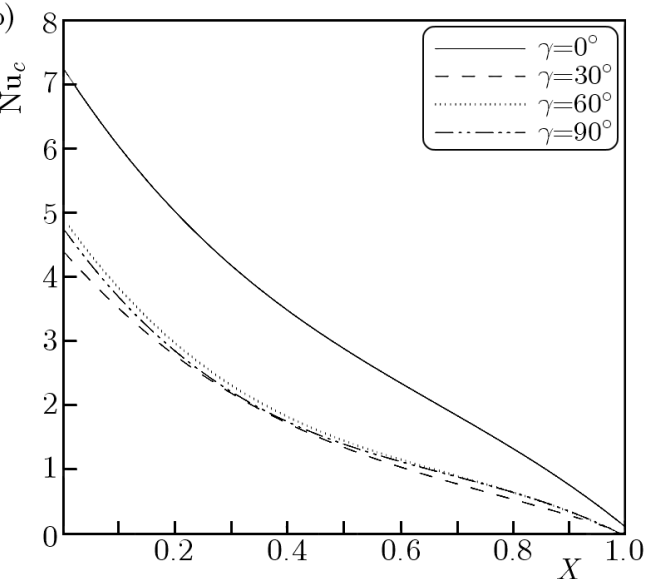

Fig. 5. Local Nusselt number distributions for different counter-clockwise inclination angles, Ri $=1$, Gr $=10^{4}$, and $A=1$; (a) along the hot wall, (b) along the cold wall

Figures 5a and 5b show the variations of the local Nusselt number along the hot and the cold walls of the cavity for $\mathrm{Ri}=1$ and for different counter-clockwise inclination angles, respectively. 
Moving from the left to the right along the hot wavy wall, it is noticed that the local Nusselt number increases in the vicinity of the first hill by increasing the inclination angle (Fig. 5a). This increase in the local Nusselt number is attributed to the enhancement of the natural convection, and the resulting boundary layer formed along the hot wavy wall. Along the remaining portions of the wavy wall, the local Nusselt number decreases substantially with an increase in the inclination angle (Fig. 5a). The reduction of the Nusselt number is due to the fact that the natural convection along the wavy wall in these regions opposes the primary recirculation vortex. Moreover, as it can be observed from Fig. 5b, the local Nusselt number along the cold wall decreases with an increase in the inclination angle which is attributed to the shrinkage of the primary vortex.

The streamlines and the isotherms inside the cavity for different inclination angles obtained by tilting the cavity clockwise are displayed in Figs. 6a and 6b, respectively. The results in these figures are obtained for $\mathrm{Ri}=1, \mathrm{Gr}=10^{4}$, and $A=1$. By tilting the cavity clockwise about point $O$ (Fig. 1), the natural convection along the hot wavy wall, unlike the counter-clockwise case, assists the primary recirculation vortex (Fig. 6a). This, in turn, would result in the enhancement of temperature gradients along the cold and the hot walls of the cavity (Fig. 6b). Therefore, the local Nusselt numbers along the hot and the cold cavity walls, which are depicted in Figs. $7 \mathrm{a}$ and 7b, respectively, increase with an increase in the inclination angle.

(a) Streamlines

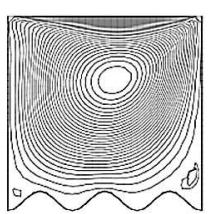

(b) Isotherms

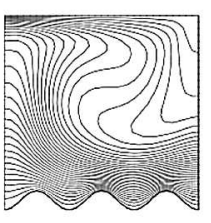

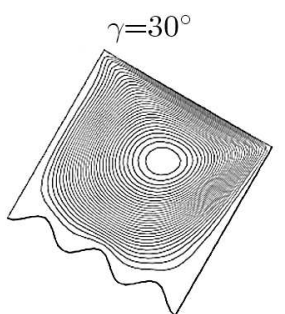

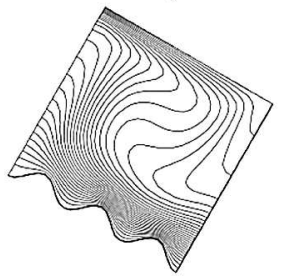

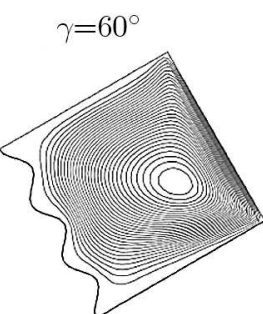

$\gamma=90^{\circ}$
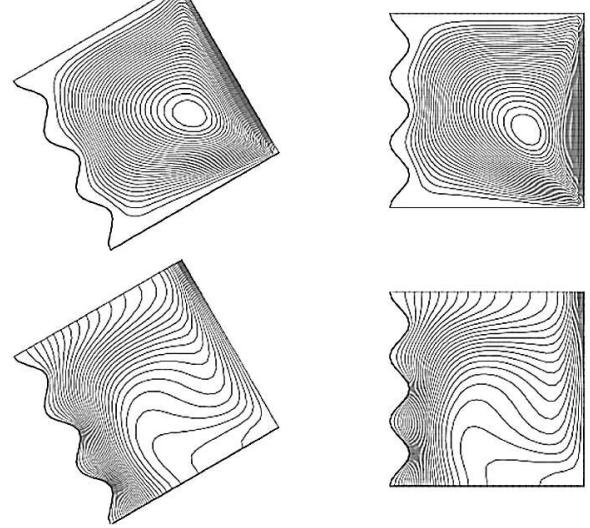

Fig. 6. Streamlines and isotherms for different clockwise inclination angles, $\mathrm{Ri}=1, \mathrm{Gr}=10^{4}$, and $A=1$; (a) streamlines, (b) isotherms

(a)

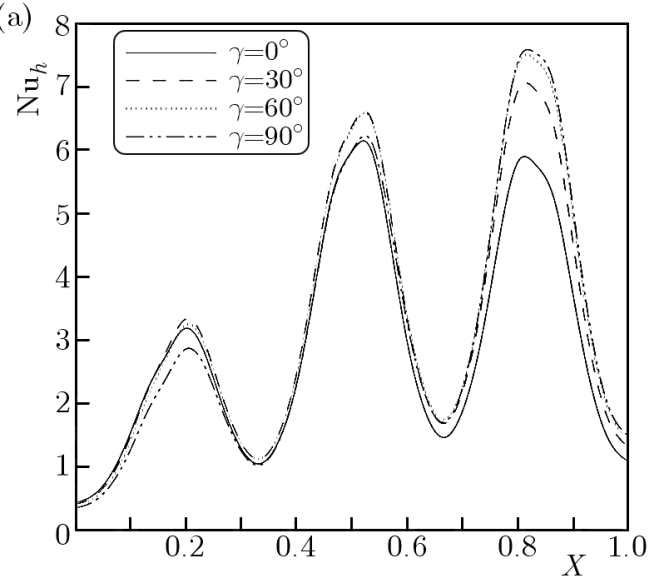

(b)

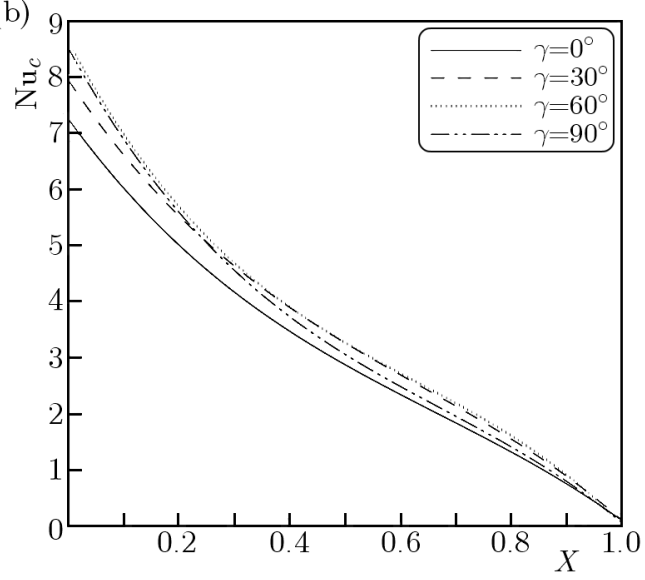

Fig. 7. Local Nusselt number distributions for different clockwise inclination angles, $\mathrm{Ri}=1, \mathrm{Gr}=10^{4}$, and $A=1$; (a) along the hot wall, (b) along the cold wall 
Figures $8 \mathrm{a}$ and $8 \mathrm{~b}$ show the variations of the average Nusselt number with the inclination angle for tilting the cavity counter-clockwise and clockwise about point $O$ (Fig. 1), respectively. The results in these figures are for $\mathrm{Ri}=1, \mathrm{Gr}=10^{4}$, and $A=1$. It is observed in Fig. 8a that the optimal angle which provides the highest average Nusselt number while tilting the enclosure counter-clockwise is $\gamma=0^{\circ}$, i.e. the horizontal cavity. However, the optimal angle while tilting the cavity clockwise is about $\gamma=60^{\circ}$ (Fig. 8b).
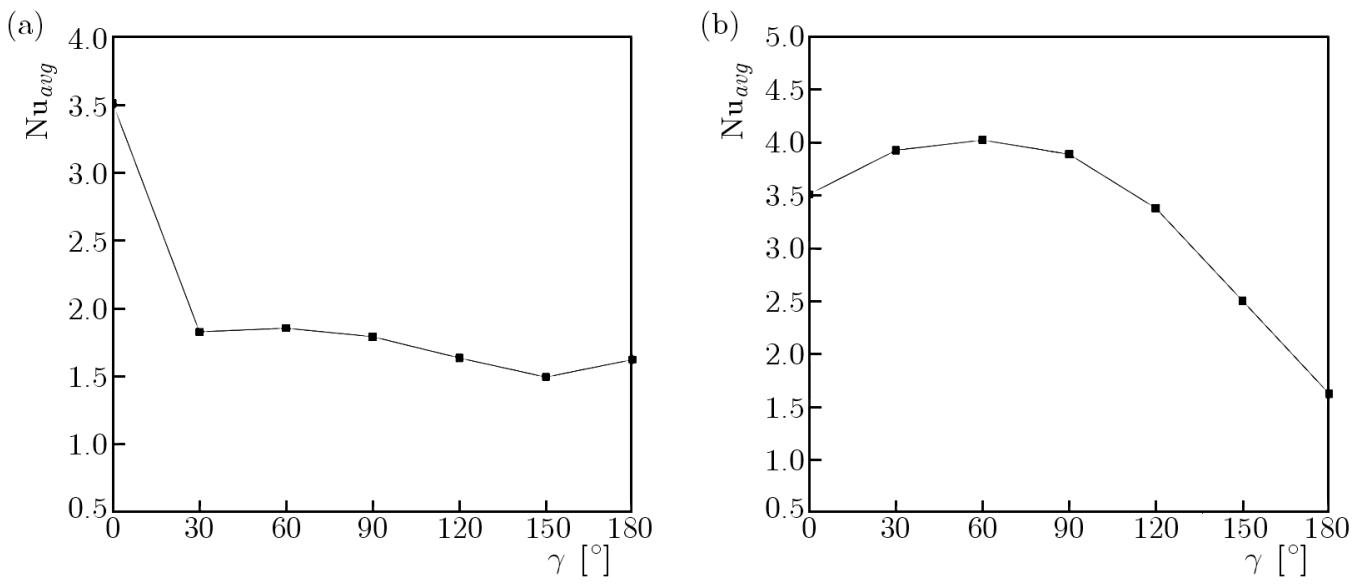

Fig. 8. Variation of the average Nusselt number with the inclination angle for $\mathrm{Ri}=1, \mathrm{Gr}=10^{4}$, and $A=1$; (a) tilting counter-clockwise, (b) tilting clockwise

As far as the effects of the aspect ratio of the cavity on the fluid flow and heat transfer behavior are concerned, Figures $9 \mathrm{a}$ and $9 \mathrm{~b}$ show the streamlines and the isotherms inside the cavity, respectively. The figures are obtained for the aspect ratios of $0.5,1$, and 2 while keeping $\mathrm{Ri}=1$ and $\mathrm{Gr}=10^{5}$. As it can be observed from Fig. 9a, the DSE enhances with the increasing aspect ratio of the cavity, and a relatively large counter-clockwise eddy is generated in this region (Fig. 9a). In fact, for the aspect ratio equal to two, the effect of the forced convection does not reach the bottom of the cavity. Therefore, the temperature gradient decreases in the vicinity of the hot wavy wall with increasing the aspect ratio of the cavity (Fig. 9b). As a result, the average Nusselt number of the hot wall of the cavity decreases from 6.3855 (for the aspect ratio of 0.5 ) to 4.5281 for the aspect ratio of 1 , and to 2.6750 for the aspect ratio of 2 .

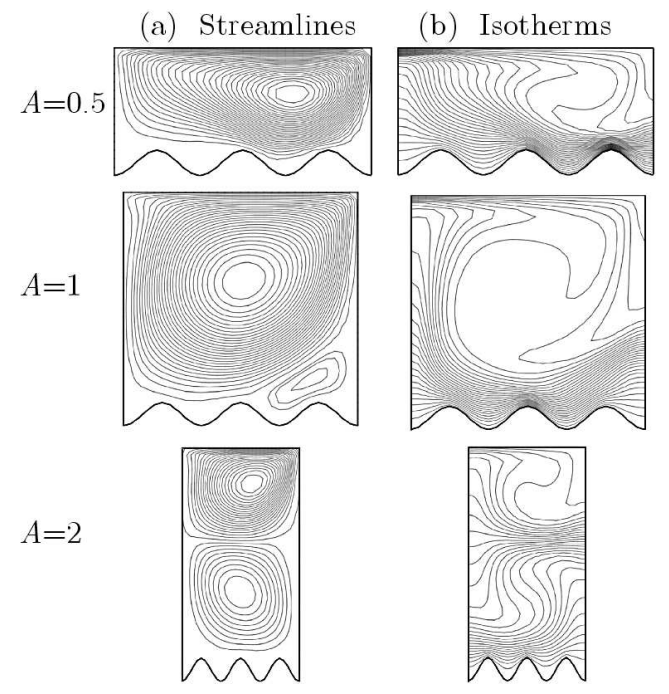

Fig. 9. Streamlines (a) and isotherms (b) inside the cavity for different aspect ratios, $\gamma=0, \mathrm{Ri}=1$, and $\mathrm{Gr}=10^{5}$ 


\section{Conclusions}

The MLPG method is extended to numerically investigate the incompressible steady laminar flow of a fluid in an irregular geometry domain sustaining mixed convection heat transfer. The forced convective flow within the cavity is attained by a sliding top lid, while the natural convective effect is obtained by subjecting the bottom wavy wall to a higher temperature than its top sliding counterpart. The attention on this present study stems from furthering the applicability of the MLPG method by focusing on, perhaps, the most important mode of the heat transfer, the mixed convection for which the broadest spectra of engineering, technological and natural applications exist. The results show that the clockwise inclination angle tilting assists the primary recirculation vortex, causing the local Nusselt number for both the hot and cold walls to increase. The optimal inclination angle to achieve the maximum heat transfer is about $60^{\circ}$. Also, for zero inclination angle, the results show that a decrease in the aspect ratio enhances the hot wall average Nusselt number as the effect of the forced convection on the hot wall increases for the shorter cavity.

\section{References}

1. Al-Amiri A., Khanafer K., Bull J., Pop I., 2007, Effect of the sinusoidal wavy bottom surface on mixed convection heat transfer in a lid-driven cavity, International Journal Heat and Mass Transfer, 50, 1771-1780

2. Arefmanesh A., Najafi M., Abdi H., 2005, A meshless local Petrov-Galerkin method for fluid dynamics and heat transfer applications, Journal of Fluids Engineering, 127, 647-455

3. Arefmanesh A., Najafi M., Abdi H., 2008, Meshless local Petrov-Galerkin method with unity test function for non-isothermal fluid flow, Computer Modeling in Engineering and Sciences, 25, $9-23$

4. AtuUri S.N., Zhu T., 1998, A new meshless local Petrov-Galerkin (MLPG) approach in computational mechanics, Computational Mechanics, 22, 117-127

5. Atudri S.N., Zhu T., 1998, A new meshless local Petrov-Galerkin (MLPG) approach to nonlinear problems in computer modeling and simulation, Computer Modeling in Engineering and Sciences, 3, 187-196

6. Bejan A., 2004, Convection Heat Transfer, John Wiley \& Sons, New York

7. Chamkна A.J., 2002, Hydromagnetic combined convection flow in a vertical lid-driven cavity with internal heat generation or absorption, Numerical Heat Transfer, Part A, 41, 529-546

8. Guo G., Sharif M.A.R., 2004, Mixed convection in rectangular cavities at various aspect ratios with moving isothermal sidewalls and constant flux heat source on the bottom wall, International Journal of Thermal Science, 43, 465-475

9. Haji Mohammadi M., 2008, Stabilized meshless Petrov-Galerkin method (MLPG) for incompressible viscous fluid flows, Computer Modeling in Engineering and Sciences, 29, 75-94

10. Khanafer K., Al-Amiri A.M., Pop I., 2007, Numerical simulation of unsteady mixed convection in a driven cavity using an externally excited sliding lid, European Journal of Mechanics - B/Fluids, 26, 669-687

11. Lin H., Atluri S.N., 2000, Meshless local Petrov-Galerkin (MLPG) method for convectiondiffusion problems, Computer Modeling in Engineering and Sciences, 1, 45-60

12. Lin H., Atluri S.N., 2001, The meshless Local Petrov-Galerkin (MLPG) method for solving incompressible Navier-Stokes equations, Computer Modeling in Engineering and Sciences, 1, $117-142$ 
13. Moallemi M.K, JAng K.S., 1992, Prandtl number effects on laminar mixed convection heat transfer in a lid-driven cavity, International Journal Heat and Mass Transfer, 35, 1881-1892

14. NASRIN R., 2012, Influences of physical parameters on mixed convection in a horizontal lid-driven cavity with an undulating base surface, Numerical Heat Transfer, Part A, 61, 306-321

15. Onate E., Idelsohn S., Zienkiewicz O.Z., Taylor R.L., 1996, A finite point method in computational mechanics: applications to convective transport and fluid flow, International Journal for Numerical Methods in Engineering, 39, 3839-3867

16. Oztop H.F., Dagtekin I., 2004, Mixed convection in two-sided lid-driven differentially heated square cavity, International Journal Heat and Mass Transfer, 47, 1761-1769

17. Sharif M.A.R., 2007, Laminar mixed convection in shallow inclined driven cavities with hot moving lid on top and cooled from bottom, Applied Thermal Engineering, 27, 1036-1042

18. Zhu T., Zhang J.D., Atluri S.N., 1998, Local boundary integral equation (LBIE) for solving nonlinear problems, Computational Mechanics, 22, 174-186

Manuscript received June 7, 2014; accepted for print September 5, 2014 